\title{
Case Report \\ Role of Bedside Ultrasound in CMV Retinitis: A Case Report
}

\author{
Lauren Westafer, ${ }^{1}$ L. Connor Nickels, ${ }^{2}$ Eike Flach, ${ }^{2}$ \\ Giuliano De Portu, ${ }^{2}$ and Latha Ganti Stead ${ }^{2}$ \\ ${ }^{1}$ College of Osteopathic Medicine, Nova Southeastern University, Fort Lauderdale, FL 33314, USA \\ ${ }^{2}$ Department of Emergency Medicine, University of Florida College of Medicine, Gainesville, FL 32610, USA
}

Correspondence should be addressed to Latha Ganti Stead, lstead@ufl.edu

Received 11 October 2012; Accepted 30 October 2012

Academic Editors: L. Bojić, C.-C. Lai, and H. P. Wu

Copyright (C) 2012 Lauren Westafer et al. This is an open access article distributed under the Creative Commons Attribution License, which permits unrestricted use, distribution, and reproduction in any medium, provided the original work is properly cited.

We present a case of retinal detachment diagnosed by emergency department bedside ultrasonography in a patient with CMV retinitis. The indications and findings of ocular ultrasonography are discussed.

\section{Introduction}

Retinal detachments, though uncommon, are devastating ocular emergencies that may result in permanent vision loss. While most retinal detachments are associated with age, myopia, inflammatory disorders, and trauma, individuals with human immunodeficiency virus (HIV) are at risk for CMV retinitis and subsequent detachment [1]. Individuals with CMV retinitis have an incidence of retinal detachment of approximately $50 \%$ per patient per year, as a result of the virus-mediated necrosis of the retina [2]. Although early detection of retinal detachment may preserve a patient's vision, CMV retinitis can infect both eyes and often progress to retinal detachment in days to weeks. As a result, practitioners should maintain a high index of suspicion for retinal detachment in HIV positive patients and use bedside ultrasound as a means of expedient evaluation in patients with visual changes.

\section{Case}

A 38-year-old male presented to the Emergency Department (ED) with a complaint of progressive vision loss in his left eye over the past week. The patient denied trauma and any prior ocular history. He denied photophobia, pain, discharge, pruritus, nausea, vomiting, or headache.

Physical exam revealed a pleasant, well-developed male who appeared comfortable. His past medical history was significant for HIV with an unknown CD4+ cell count.
He was not on antiretroviral therapy but was undergoing treatment for toxoplasmosis. On exam, he had no facial swelling, erythema, or discharge from his eyes. His conjunctivas were injected bilaterally. His extraocular movements were intact. The patient's pupils were round bilaterally, but his left pupil was nonreactive to light. There was no pain with movement of his extra-ocular muscles. He reported complete loss of vision in his left eye, including inability to perceive light. Visual acuity in the patient's right eye was $20 / 200$, his reported baseline.

A high frequency $7.5-10-\mathrm{MHz}$ linear array transducer was used to perform the ocular examination. A large amount of standard, water-soluble ultrasound gel was applied to the patient's closed eyelid. The patient was instructed to look straight ahead. The eye was scanned in both the sagittal and transverse planes, using essentially no pressure on the globe. The ultrasound demonstrated a large retinal detachment in the left eye with no macular sparing (Figure 1). No vitreous hemorrhage was detected, and findings of elevated intracranial pressure were not present (Figure 2). Ultrasound of the right eye demonstrated no retinal detachment and a normal globe. Ophthalmology was consulted and asked to see the patient in the outpatient clinic. The patient was diagnosed with CMV retinitis in his bilateral eyes. He was treated with intravitreal Foscarnet, Pred-Forte (prednisolone acetate) eye drops, and Vigamox eye drops. At two-week followup, vision in his right eye was improving, and no retinal detachment had developed in that eye. 


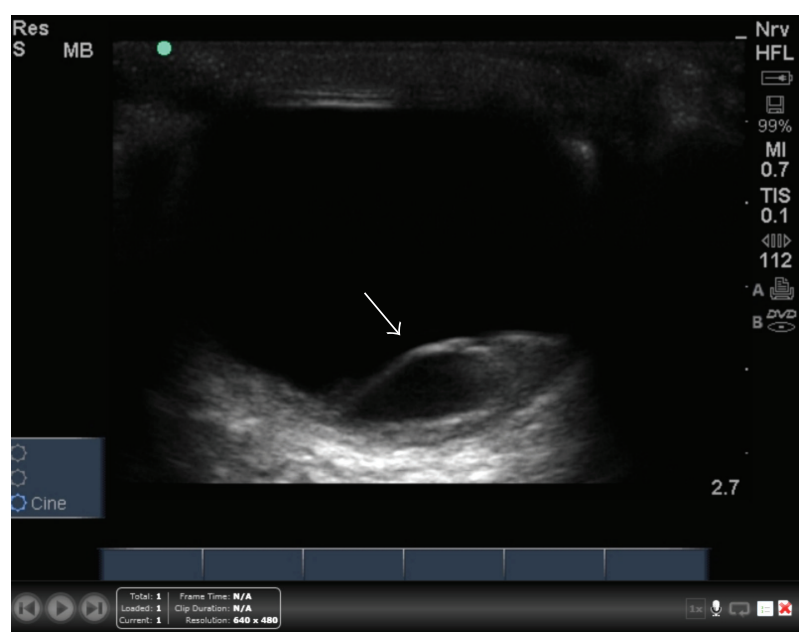

FIGURE 1: Left eye ultrasound shows retinal detachment.

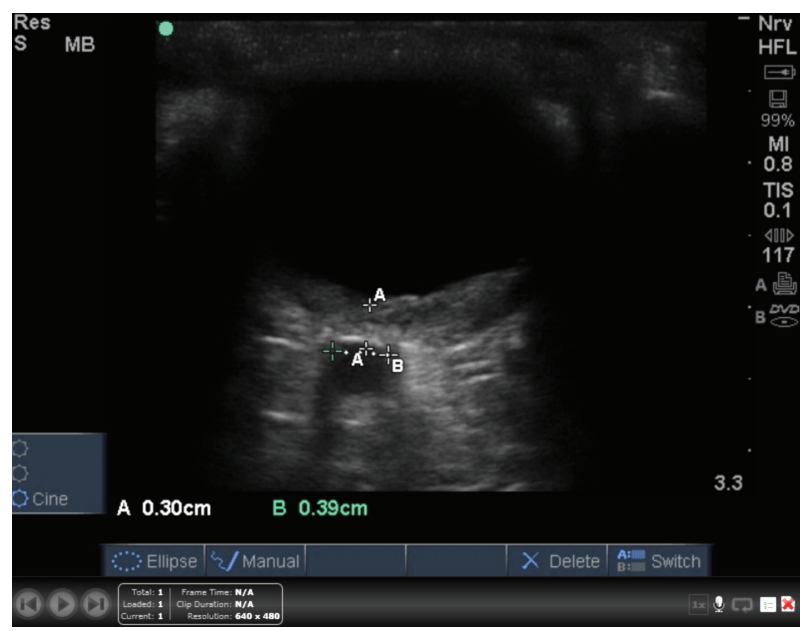

FIGURE 2: Left eye optic nerve sheath measurement, consistent with normal ICP. (Below).

\section{Discussion}

Retinal detachment lacks obvious physical findings and may result in monocular blindness, especially if missed. Historically, the gold standard diagnostic examination for retinal detachment is a thorough ophthalmoscopic evaluation, but this is often not immediately feasible due to consultant availability, dilation contraindications and equipment, or medication limitations $[3,4]$.

Ophthalmologists have used ultrasound for decades. Retinal detachment can be described as a thick, hyperechoic membrane that appears dissociated from the posterior segment of the eye. This membrane is often described as undulating and may be difficult to detect if the retinal detachment is small [4].

Emergency physicians have been using ultrasound to correctly diagnose retinal detachment for over a decade [5]. Recently, more studies have validated the limited training requirements needed for emergency physicians to accurately detect retinal detachment $[6,7]$. Studies vary, with reports of twenty minutes to one-hour lectures followed by handson practicums. As a result, bedside ultrasound is gaining momentum in the diagnosis of this condition. Prospective studies in the Emergency Department have demonstrated that emergency physicians have $97-100 \%$ sensitivity in diagnosing retinal detachment using ultrasound. The specificity is not compromised, with studies reporting specificities of $92 \%[4,8,9]$. The literature also has shown that bedside ultrasonography has been able to correlate nerve sheath diameter (ONSD) with abnormalities in intracranial pressure [10].

The most emergent retinal detachments are when the area of the macula is not detached, termed a "mac-on" retinal detachment [5]. Detached retinas that preserve the area of the macula can benefit from early intervention and allow the patient to regain their predetachment vision. Unfortunately, delays in diagnosis and intervention result in a progressive condition that eventually involves the macula, leaving the patient with a permanent deficit [11]. Use of bedside ultrasound allows emergency physicians to quickly diagnose and risk-stratify patients with a potential ocular emergency. In the case of our patient, the vision in his right eye was preserved as a result of detection and treatment. Evaluation of the ONSD showed a $3.9 \mathrm{~mm}$ measurement consistent with normal ICP [10]. Furthermore, emergency physicians detecting a "mac-on" retinal detachment may be able to advocate for quicker evaluation by ophthalmology or transfer to a facility capable of ophthalmologic intervention. Further investigation is needed to determine the ability of emergency physicians to differentiate between "mac-on" and "mac-off" detachments.

\section{References}

[1] J. Arroyo, "Retinal Detachment," Uptodate. July 18, 2012, http://www.uptodate.com.ezproxylocal.library.nova.edu/contents/retinal-detachment?source=search_result\&search=retinal+detachment\&selectedTitle $=1 \sim 118$.

[2] J. H. Kempen, D. A. Jabs, J. P. Dunn, S. K. West, and J. Tonascia, "Retinal detachment risk in cytomegalovirus retinitis related to the acquired immunodeficiency syndrome," Archives of Ophthalmology, vol. 119, no. 1, pp. 33-40, 2001.

[3] J. Mitchell, "Ocular emergencies," in Emergency Medicine: A Comprehensive Study Guide, J. E. Tintinalli, G. D. Kelen, J. S. Stapczynski et al., Eds., American College of Emergency Physicians, Dallas, Tex, USA, 2004.

[4] R. Yoonessi, A. Hussain, and T. B. Jang, "Bedside ocular ultrasound for the detection of retinal detachment in the emergency department," Academic Emergency Medicine, vol. 17, no. 9, pp. 913-917, 2010.

[5] N. Teismann, S. Shah, and A. Nagdev, "Focus on: ultrasound for acute retinal detachment," ACEP News, May 2009, http://apps.acep.org/publications.aspx?LinkIdentifier=id\&id= 45426\&fid 77738\&Mo=No\&taxid $=1$.

[6] M. Blaivas, D. Theodoro, and P. R. Sierzenski, "A study of bedside ocular ultrasonography in the emergency department," Academic Emergency Medicine, vol. 9, no. 8, pp. 791-799, 2002.

[7] J. Elia and R. Borger, "Diagnosis of retinal detachment in the ED with ultrasonography," Journal of Emergency Medicine, vol. 37, no. 4, pp. 415-416, 2009. 
[8] M. Blaivas, D. Theodoro, and P. R. Sierzenski, "A study of bedside ocular ultrasonography in the emergency department," Academic Emergency Medicine, vol. 9, no. 8, pp. 791-799, 2002.

[9] Z. Shinar, L. Chan, and M. Orlinsky, "Use of ocular ultrasound for the evaluation of retinal detachment," Journal of Emergency Medicine, vol. 40, no. 1, pp. 53-57, 2011.

[10] V. S. Tayal, M. Neulander, H. J. Norton, T. Foster, T. Saunders, and M. Blaivas, "Emergency Department Sonographic measurement of optic nerve sheath diameter to detect findings of increased intracranial pressure in adult head injury patients," Annals of Emergency Medicine, vol. 49, no. 4, pp. 508-514, 2007.

[11] R. F. Gariano and C. H. Kim, "Evaluation and management of suspected retinal detachment," American Family Physician, vol. 69, no. 7, pp. 1691-1698, 2004. 


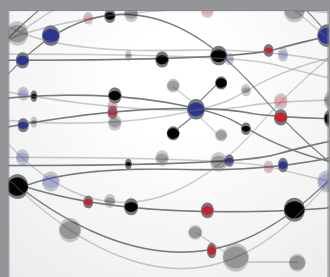

The Scientific World Journal
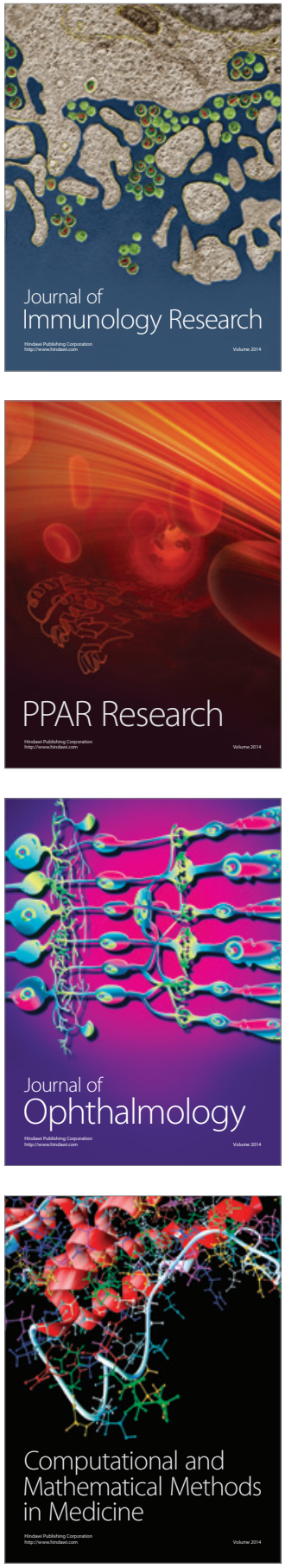

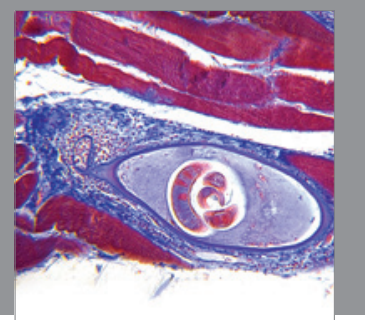

Gastroenterology

Research and Practice
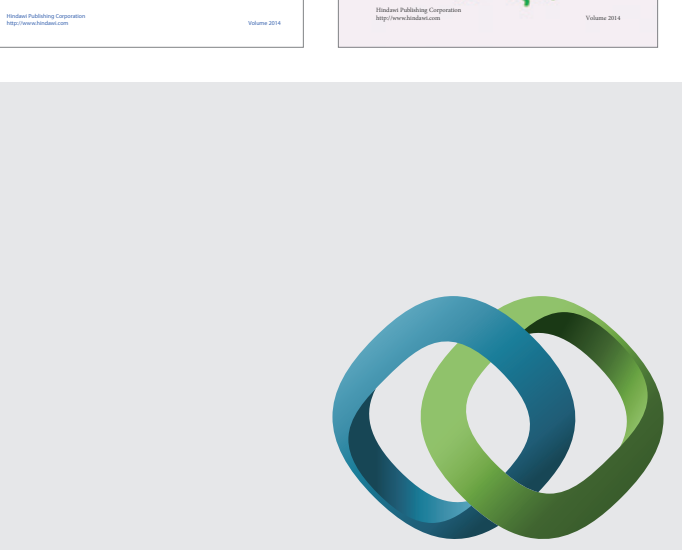

\section{Hindawi}

Submit your manuscripts at

http://www.hindawi.com
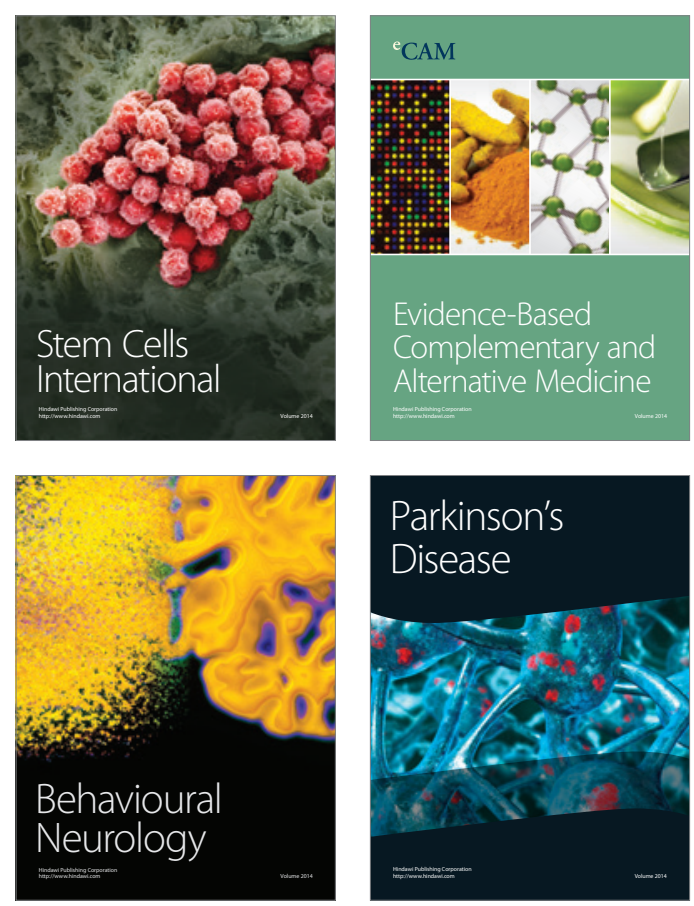

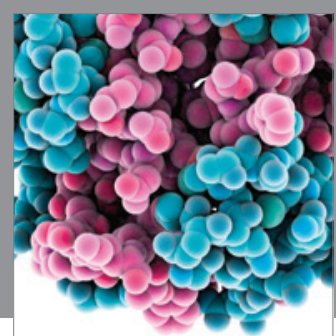

Journal of
Diabetes Research

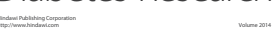

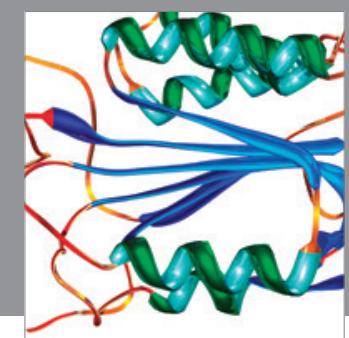

Disease Markers
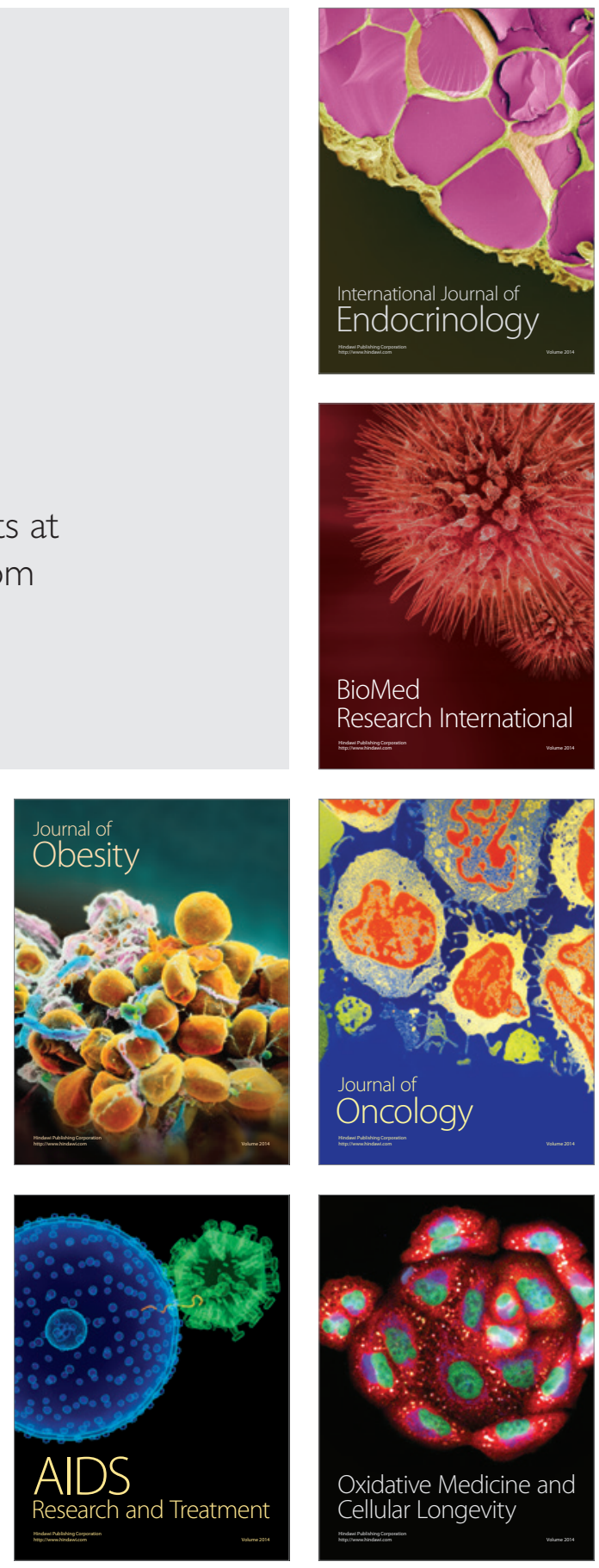